\title{
ORAL SWITCH OF CONTINUOUS INTRAVENOUS MORPHINE IN VENTILATED VERY PREMATURE INFANTS: A RETROSPECTIVE STUDY ON EFFICACY, EFFICIENCY AND TOLERANCE
}

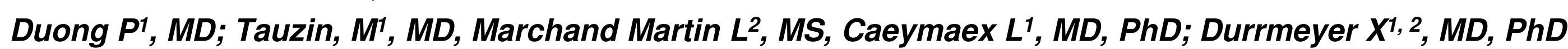

1: Neonatal Intensive Care Unit, CHI Créteil, Créteil, Université Paris Est Créteil, France

2: INSERM U1153, Obstetrical, Perinatal and Pediatric Epidemiology Team, CRESS, Paris Descartes University, France

Contacts : phoi.duong@chicreteil.fr; xavier.durrmeyer@chicreteil.fr

\section{Background}

Continuous intravenous (IV) morphine is commonly used in ventilated neonates ${ }^{1}$. Oral route is theoretically feasible but data on oral morphine in ventilated premature infants are lacking. Most data on oral morphine currently available address neonatal abstinence syndrome.

\section{Objective}

After implementing a protocol for oral switch of IV continuous morphine in ventilated neonates, we aimed at assessing the efficacy, efficiency and tolerance of this protocol

\section{Design \& Setting}

Retrospective study in a single level III center's Neonatal Intensive Care Unit.

\section{Patients}

Ventilated premature infants hospitalized in the NICU in 2016 and 2017, receiving continuous IV morphine and switched to oral morphine. We excluded patients treated for withdrawal syndrome or palliative care.

\section{Intervention - Local protocol}

In ventilated neonates receiving continuous IV morphine, the attending physician could decide to switch this treatment to the oral route if she/he expected that mechanical ventilation would last at least 3 more days and if enteral feeding was well tolerated. The oral daily dose was the same as the IV daily dose, divided in 4 doses (q6h) through a gastric tube.

\section{Outcome measures}

Pain scores (ComfortNeo scale ${ }^{2}$ ) and morphine doses were analyzed over time using Friedman's test ( a non-parametric test for repeated measures) in the 24 hours preceding and the 48 hours following the oral switch. Ventilation parameters, digestive disorders (interruption of enteral feeding), urine retention, hypotension, occurrence of withdrawal syndrome after stopping morphine and use other analgesics/sedatives were also collected.

Results

Among 44 infants who received oral morphine during the study period, 17 fulfilled inclusion criteria.

Their characteristics are summarized in the Table below.

All but one were continued on oral morphine after the switch. In 1 patient, IV morphine was resumed 42 hours after the oral switch because of high ComfortNeo scores $(=15)$.

\begin{tabular}{lc}
\hline Demographic and clinical characteristics of patients, $\mathbf{N}=17$ & \\
\hline Sex ratio (male/female) & $10 / 17$ \\
Median (IQR) gestational age at birth (weeks) & 25.9 \\
& $(24.6-26.9)$ \\
Median (IQR) Birth weight (g) & 670 \\
Small for gestational age, $\mathrm{n}$ (\%) & $(620-750)$ \\
Cause for intubation, $\mathrm{n}$ (\%) & $6(35 \%)$ \\
Respiratory distress syndrome & \\
Repeated desaturations and apnea & $7(41 \%)$ \\
Bradycardia & $8(47 \%)$ \\
Surgery & $1(6 \%)$ \\
Median (IQR) postnatal age at the time of oral switch & $1(6 \%)$ \\
(days) & 30 \\
Median (IQR) postconceptional age at the time of oral & $(22-36)$ \\
switch (weeks) & 30.4 \\
Median (IQR) weight at the time of oral switch (g) & $(27.9-31.4)$ \\
\hline
\end{tabular}

Results (cont'd)

No significant change over time was observed for ComfortNeo scores $(\mathrm{p}=0.15)$ (Fig. 1).

Median (IQR) morphine doses were $13.5(10-20) \mu \mathrm{g} / \mathrm{kg} / \mathrm{h}$ in the IV period and significantly increased to $15(10-25) \mu \mathrm{g} / \mathrm{kg} / \mathrm{h}$ in the oral period ( $p=0.009)$ (Fig. 2).

Eleven patients received benzodiazepine during the IV morphine period and 12 during the oral morphine period.

No significant change over time was observed for mean airway pressures $(p=0.13)$ with median [IQR] values of 12.4 [10.7-13] and $10.6[9.1-12.5] \mathrm{cm} \mathrm{H}_{2} \mathrm{O}$ for the periods before and after the oral switch, respectively.

No significant change over time was observed for $\mathrm{FiO}_{2}(\mathrm{p}=0.24)$.

No digestive or urinary adverse event was observed. After a median (IQR) duration of oral morphine treatment of 13 (4-20) days, 11 (65\%) patients showed signs of withdrawal.

Figure 1

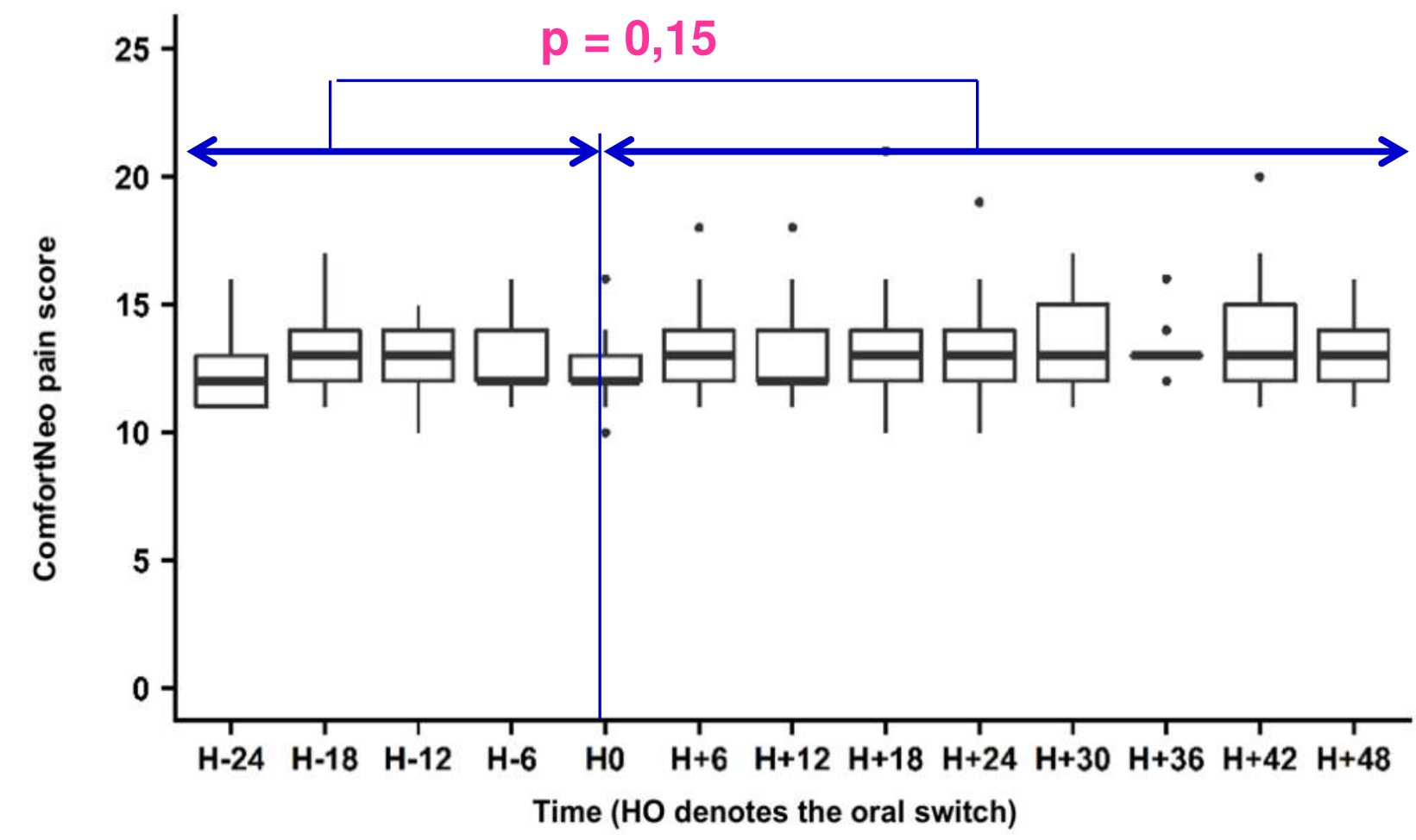

Figure 2

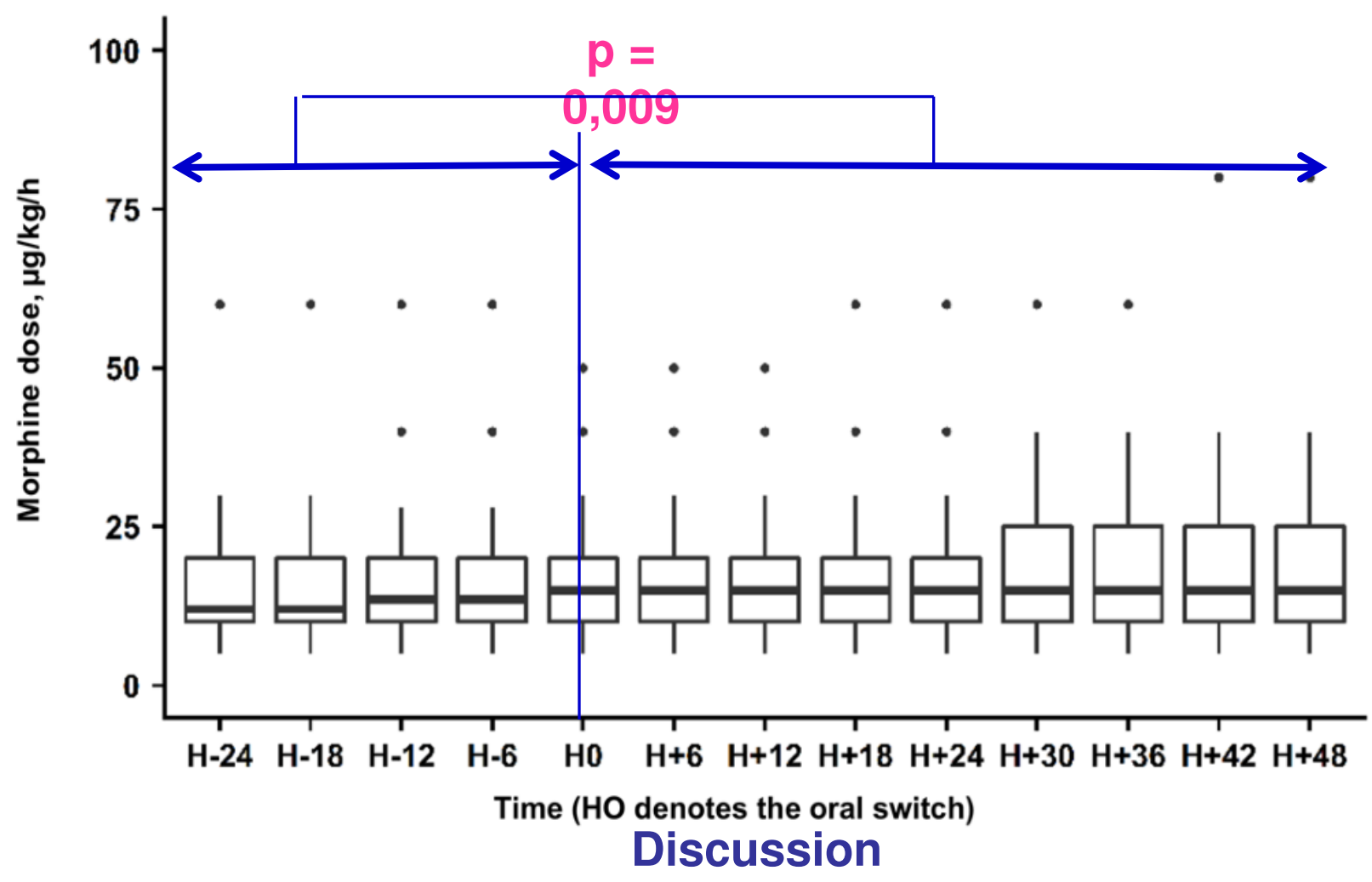

First report of oral morphine use in ventilated very premature infants.

Oral bioavailability might be higher in preterm than in term infants ${ }^{3}$ (mild increase in doses as compared to IV route).

However, increased doses at oral switch might be more effective.

Small sample, no long term assessment, no PK data

\section{Conclusion}

Oral morphine is a feasible alternative to IV morphine in ventilated neonates in the NICU but deserves further studies.

References

1: Carbajal R et al. Lancet Respir Med. 2015 Oct;3(10):796-812 2: van Dijk M et al. Clin J Pain. 2009 Sep;25(7):607-16. 3: Liu T et al. J Clin Pharmacol. 2016;56(8):1009-18. 